\title{
Resultados clínicos e radiográficos de placas ósseas bloqueadas em 13 casos
}

\author{
Clinical and radiographics results of locking plates in 13 cases
}

\section{Cássio Ricardo Auada FERRIGNO ${ }^{1}$; Olicies da CUNHA ${ }^{1}$; Daniela Fabiana Izquierdo CAQUIAS ${ }^{1}$; Kelly Cristiane ITO $^{1}$; Marcos Ishimoto DELLA NINA ${ }^{1}$; Tatiana Casimiro MARIANI ${ }^{1}$; Vanessa Couto de Magalhães FERRAZ1}

${ }^{1}$ Departamento de Cirurgia da Faculdade de Medicina Veterinária e Zootecnia da

Universidade de São Paulo, São Paulo - SP, Brasil

\begin{abstract}
Resumo
A Placa Óssea Bloqueada (POB) consiste em um novo sistema de fixação interna, onde a placa apresenta orifícios duplos, um liso para compressão e outro rosqueado para fixação do parafuso que se fixa à placa. Promove grande estabilidade à fratura, sendo possível associar parafusos neutros e compressivos. Parafusos tradicionais comprimem a placa ao osso, nas placas bloqueadas não existe esta força e o encaixe da cabeça do parafuso à placa resulta em menores danos ao suporte vascular periosteal. Treze cães com distúrbios ortopédicos diversos foram tratados cirurgicamente com placa bloqueada com resultados satisfatórios. A placa bloqueada pode ser utilizada na medicina veterinária, porém, é técnica que requer cuidado e planejamento pré-operatório, especialmente na sequência de aplicação dos diferentes tipos de parafusos. Apresenta custo elevado, mas confere estabilidade rígida do foco de fratura e minimiza a possibilidade de perda prematura da interface parafuso e osso, diminuindo a possibilidade de instabilidade precoce e soltura do implante.
\end{abstract}

Palavras-chave: Placas bloqueadas. Cães. Osteotomia. Osteossíntese.

\begin{abstract}
Locking plate is a new internal fixation system where the plate features has double holes, one smooth for compression and other threaded for screw fixing that attaches the plate. It leads to greater stability to the fracture being possible to associate neutral and compressive screws. Traditional screws compress the plate to the bone, in the locking plates there are no such strength and the fit of the screw head to the plate results in less damage to the vascular periosteal support. Thirteen dogs with various orthopedic disorders were treated surgically by locking plate with satisfactory results. The locking plate can be used in veterinary medicine, however, it is a technique that requires care and preoperative planning, especially in the order of applying different types of screws. It is expensive, but provides rigid stability of the fracture site and minimizes the likelihood premature loss of the screw and bone interface, reducing the possibility of instability and early loosening of the implant.
\end{abstract}

Keywords: Locking plate. Dogs. Osteotomy. Osteosynthesis.

\section{Introdução}

As pesquisas em ortopedia tem se preocupado com abordagens mais fisiológicas e mínimos distúrbios dos tecidos moles em reconhecimento à importância de fatores biológicos na fixação interna e consolidação da fratura. Em resposta a esta tendência, novas gerações de implantes foram desenvolvidas ${ }^{1,2}$.

A Placa Óssea Bloqueada (POB) consiste em um novo sistema de fixação interna destinado a limitar o contato entre o implante e o osso com objetivo de minimizar o prejuízo do suprimento vascular para o osso. A POB pode apresentar orifícios únicos (para bloqueio) ou orifícios duplos, sendo um liso para compressão e outro rosqueado para fixação do parafuso que fixa a placa. Promove estabilidade da fratura

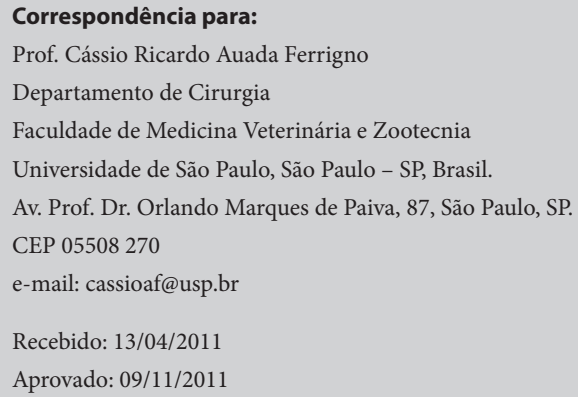


sendo possível associar parafusos neutros e compres$\operatorname{sivos}^{2}$. As extremidades da placa têm formato cônico, que permite a introdução sob a musculatura, em procedimentos minimamente invasivos. Parafusos tradicionais comprimem a placa ao osso, nas placas bloqueadas, não existe esta força e o encaixe da cabeça do parafuso à placa resulta em menores danos ao suporte vascular periosteal, importante para consolidação ${ }^{3}$. A mínima distância entre a placa e o osso dificulta a formação de biofilme, podendo ser usada em casos de osteomielites. Desta forma, promove grande estabilidade e permite a consolidação mesmo em casos de infecções ${ }^{2,4}$.

No sistema POB, a interface parafuso/placa impede movimentação entre as partes individuais do componente. Assim, todos os parafusos em um fragmento devem se soltar para a estabilidade ser perdida. Este novo conceito é chamado de estabilidade angular, onde os parafusos, por estarem presos à placa, não mudam sua relação em ângulo com o implante. Em contrapartida, no sistema de placa convencional, a estabilidade depende da compressão e atrito entre a placa e a superfície óssea. Assim, a estabilidade do componente é diminuída ou perdida quando um ou mais parafusos se solta ${ }^{2}$.

O sistema de $\mathrm{POB}$ é comumente usado para reparação de fraturas em pacientes humanos, sobretudo aqueles com fragilidade óssea ${ }^{5}$. Estudo recente demonstrou resultados semelhantes, no que se refere à consolidação óssea, em 47 pacientes humanos tratados com POB ou haste intramedular bloqueada ${ }^{6}$. Testes mecânicos mostram eficiência das POB em rádio ${ }^{7}$, constituindo uma alternativa eficaz no tratamento de luxações rádio-cárpicas.

Um estudo comparou o sistema POB com placas de compressão dinâmica, em 36 rádios osteoporóticos de cadáveres humanos. Os espécimes foram submetidos a ensaios não destrutivos de flexão e torção. Os resultados indicaram que o sistema POB é mais estável do que o sistema de placas de compressão dinâmicas ${ }^{7}$. As
POB, por interferirem pouco na vascularização, permitem consolidação rápida ${ }^{3}$. POB em fêmures também foram usadas com sucesso por Oh et al. ${ }^{8}$. A ausência de contato da placa com o osso interfere pouco na vascularização, facilitando o tratamento da osteomielite e a cicatrização ${ }^{3,6}$. Em termos de resistência, as $\mathrm{POB}$ se igualam as PCD, com a vantagem de interferir menos no suporte vascular, o que torna ideal para ossos com fragilidade ou casos de infecção ${ }^{9,10}$.

Aguila et al. ${ }^{9}$ e Goh et al. ${ }^{10}$ observaram comportamento biomecânico semelhante entre POB e PCD de contato mínimo, em fraturas de fêmures, em cadáveres caninos. Há relatos de fraturas de rádio e ulna e tíbia, em cães de grande porte, tratados com sucesso por meio do sistema $\mathrm{POB}^{11}$.

Vinte pacientes humanos com fraturas subtrocantéricas foram tratados com POB por técnica minimamente invasiva. A técnica proporcionou fixação estável, com alto índice de consolidação e taxa mínima de complicações ${ }^{8}$.

Apesar de Ferrigno et al. ${ }^{3}$ terem relatado os primeiros resultados da $\mathrm{POB}$, em ortopedia veterinária, no Brasil, ainda há pouca disseminação do método. Desta forma, o objetivo do presente estudo foi descrever a experiência clínica e os resultados de 13 casos tratados com o sistema POB.

\section{Material e Método}

O presente trabalho foi desenvolvido no Laboratório de Ortopedia e Traumatologia Comparada do Hospital Veterinário da Faculdade de Medicina Veterinária e Zootecnia da Universidade de São Paulo (LOTC- HOVET- FMVZ- USP). Foram selecionados treze pacientes com distúrbios ortopédicos variados, que foram submetidos à técnica de placa bloqueada (Figura 1), conforme a sequência descrita a seguir. Avaliaram-se o tempo de consolidação, complicações e eficiência da técnica nos pacientes operados. 


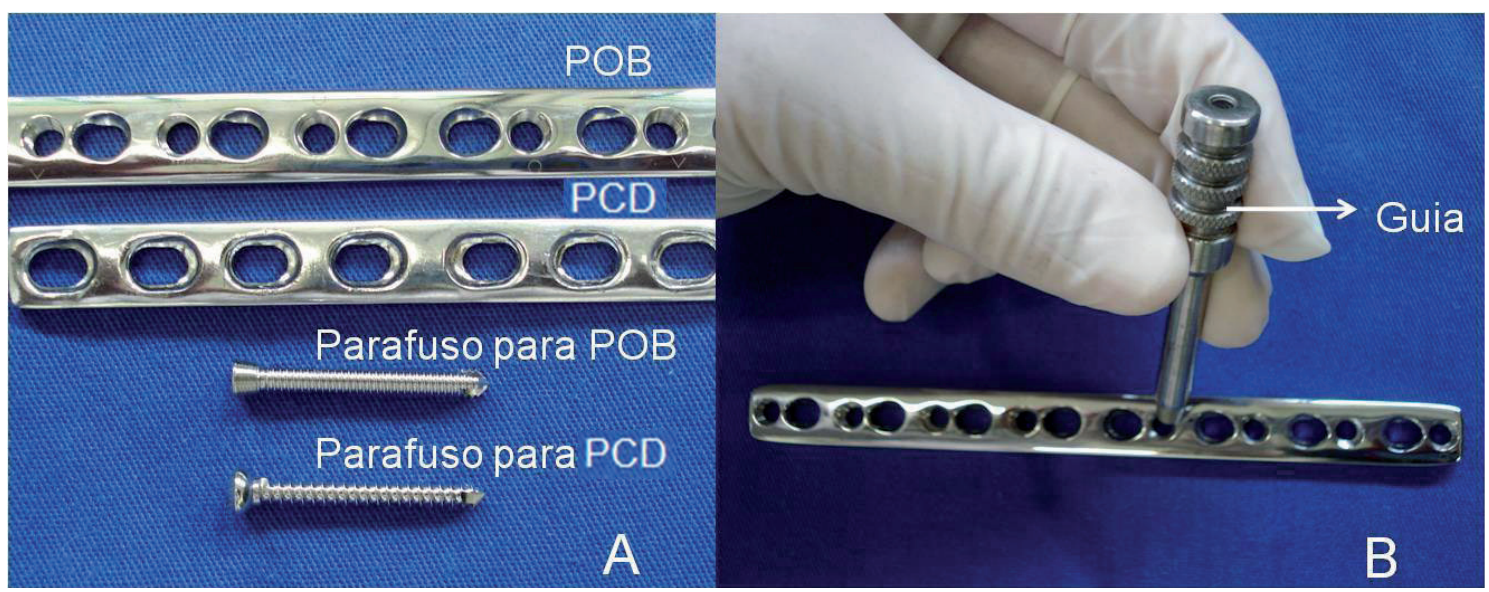

Figura 1 - Sistema de placa bloqueada e placa de compressão dinâmica. A - Placa Óssea Bloqueada (POB) e Placa de Compressão Dinâmica (PCD) com respectivos parafusos. Observe a cabeça do parafuso rosqueante da $\mathrm{POB}$ munido de rosca que se fixa no orifício circular da $\mathrm{POB}$. B - O guia da $\mathrm{POB}$ possui rosca que se fixa no orifício circular facilitando o procedimento

Os animais foram submetidos à avaliação ortopédica e, na sequência, encaminhados para radiografias em projeções médio-laterais e crânio-caudais. A técnica era planejada com base em radiografias pré-operatórias. Posteriormente foram conduzidos à cirurgia, e para tanto, adotavam-se os preparos rotineiros de campos cirúrgicos. Após abordagem indicada para a região, praticava-se o procedimento necessário e a adaptação da placa óssea bloqueada.

\section{Resumo dos casos:}

Animal 01. Cão da raça Rottweiler com $42 \mathrm{~kg}$ e nove anos de idade foi apresentado três semanas após trauma, com impotência funcional de membro torácico esquerdo. Aos exames ortopédico e radiográfico, diagnosticou-se luxação rádio-cárpica esquerda. Optou-se por artrodese com placa bloqueada com cinco parafusos no rádio, dois no carpo radial e três no terceiro metacarpo.

Animal 02. Cão sem raça definida (SRD) com 48 $\mathrm{kg}$ e oito anos de idade foi apresentado dez dias após trauma, com impotência funcional de membro torácico esquerdo. Aos exames ortopédico e radiográfico, diagnosticou-se luxação rádio-cárpica esquerda. Optou-se por artrodese com placa bloqueada com cinco parafusos no rádio, dois no carpo radial e três no terceiro metacarpo.
Animal 03. Cão da raça Pit Bull com $22 \mathrm{~kg}$ e dois anos de idade foi apresentado com luxação rádio-cárpica direita há cinco meses. Estava sendo tratada sem sucesso com penso. Usou-se placa bloqueada com três parafusos fixados no rádio, dois no carpo radial e dois no terceiro metacarpo.

Animal 04. Cão da raça Rottweiler com $50 \mathrm{~kg}$ e sete anos de idade foi apresentado com histórico de impotência funcional de membro torácico direito, há dois dias. Após diagnóstico de luxação rádio-cárpica, praticou-se artrodese do carpo com placa de 10 orifícios sendo cinco parafusos no rádio, dois no carpo radial e três no terceiro metacarpo.

Animal 05. Cão da raça Rottweiler com $40 \mathrm{~kg}$ e nove anos de idade, com histórico de queda e impotência funcional de membro torácico direito, há dois meses. Após diagnóstico de luxação rádio-cárpica, praticou-se artrodese de carpo com placa bloqueada direito, sendo cinco parafusos no rádio, dois no carpo radial e três no terceiro metatarso duas semanas. $\mathrm{O}$ procedimento foi bilateral com um mês de intervalo entre as cirurgias.

Animal 06. Cão da raça Pastor Alemão com $32 \mathrm{~kg}$ e sete anos e meio de idade foi apresentado com luxação de rádio-cárpica bilateral, há uma semana. Após 
diagnóstico clínico e radiográfico, procedeu-se artrodese bilateral com três parafusos no rádio, dois no carpo radial e dois no terceiro metacarpo (Figura 2).

Animal 07. Cão, mestiço, com $13 \mathrm{~kg}$ e sete meses de idade foi apresentado dois dias após trauma com impotência funcional de membro pélvico direito, causada por queda. Após diagnóstico de fratura transversa diafisária de fêmur, optou-se por pino intramedular e placa bloqueada com quatro parafusos proximais e três distais.

Animal 08. Cão, mestiço, com $37 \mathrm{~kg}$ e oito anos de idade, com histórico de atropelamento e impotência funcional de membro pélvico direito, há um dia. Aos exames ortopédico e radiográfico, diagnosticou-se fratura diafisária de fêmur direito. A fratura foi tratada com pino intramedular e placa bloqueada com cinco parafusos fixados no fragmento proximal e quatro no distal.

Animal 09. Cão da raça Pastor Alemão com $36 \mathrm{~kg}$ e seis anos de idade foi apresentado com histórico de fratura de fêmur direito, há dois meses, causada por atropelamento. Havia sido tratado por colega com um único pino intramedular rosqueado. $\mathrm{O}$ pino estava inserido na articulação fêmoro-tíbio-patelar, havendo osteomielite e não união óssea. $\mathrm{O}$ paciente foi submetido à osteossíntese de fêmur com placa bloqueada. Utilizaram se quatro parafusos distais e quatro proximais (Figura 2).

Animal 10. Cão, mestiço, com $42 \mathrm{~kg}$ e dois anos de idade foi apresentado com impotência funcional de membro pélvico direito, há seis meses. Diagnosticou-se luxação lateral de patela congênita com desvio angular

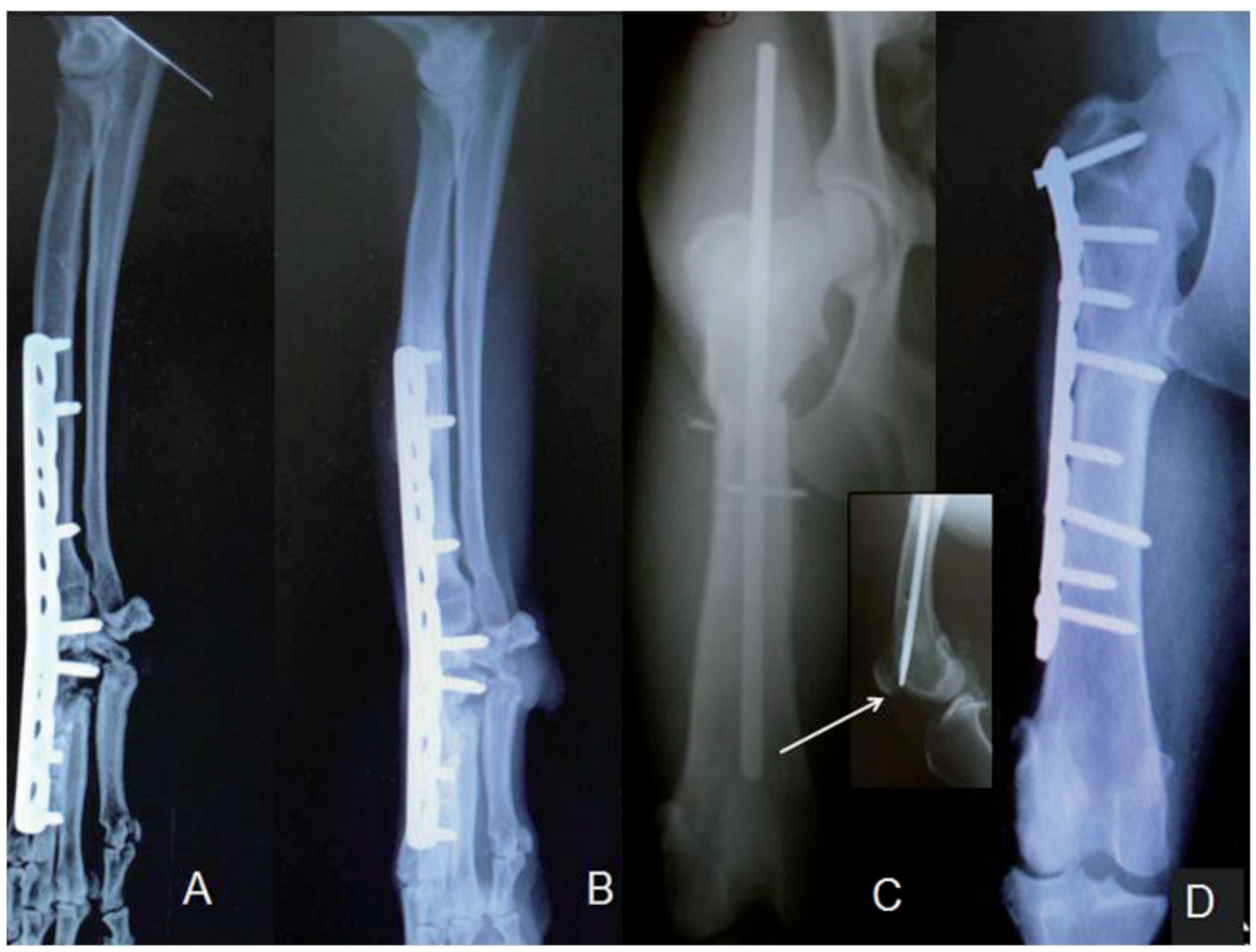

Figura 2 - Radiografias de pacientes submetidos à técnica de POB. A - POB para artrodese radiocárpica em pós-operatório imediato. B - Mesmo paciente da "Figura 2A" três meses após o procedimento. Observe consolidação. C - Fratura de fêmur com não união e pino rosqueado inserido na articulação fêmuro-tíbio-patelar (seta). D - POB para corrigir a fratura. Note parafusos monocorticais e bicorticais. Observe cicatrização óssea quatro meses após a cirurgia 
importante em fêmur distal. Para correção, procedeu-se à ostectomia em cunha, em fêmur distal, e a fratura foi estabilizada com $\mathrm{POB}$, sendo quatro parafusos proximais e dois distais. Procedeu-se à transposição da tuberosidade da tíbia para alinhamento do mecanismo do grupo muscular quadríceps.

Animal 11. Cão da raça Fila brasileiro com $14 \mathrm{~kg}$ e três meses de idade foi apresentado por incapacidade locomotora em membros pélvicos há um mês. Após exames ortopédico e radiográfico, diagnosticaram-se luxação de patela bilateral lateral grau 3 e coxa valga. Optou-se por ostectomia em cunha no fêmur e transposição da tuberosidade da tíbia, em membro pélvico esquerdo, e apenas transposição da tuberosidade da tíbia, no membro contra lateral. A ostectomia foi es- tabilizada com POB, sendo três parafusos proximais e dois distais.

Animal 12. Cão da raça Boxer com $12 \mathrm{~kg}$ e quatro meses de idade sofreu queda e foi apresentado com impotência funcional de membro pélvico esquerdo, com evolução de seis horas. Os exames físico e radiográfico demonstraram fratura em espiral de tíbia esquerda. Usou-se para estabilização $\mathrm{POB}$ com dois parafusos proximais e três distais, com técnica minimamente invasiva (Figura 3).

Animal 13. Cão, mestiço, com $26 \mathrm{Kg}$ e três anos de idade foi apresentado com histórico de impotência funcional de membro torácico esquerdo, devido a acidente com arma de fogo, há um dia. Os exames físico e radiográfico revelaram fratura distal de úmero. A fra-

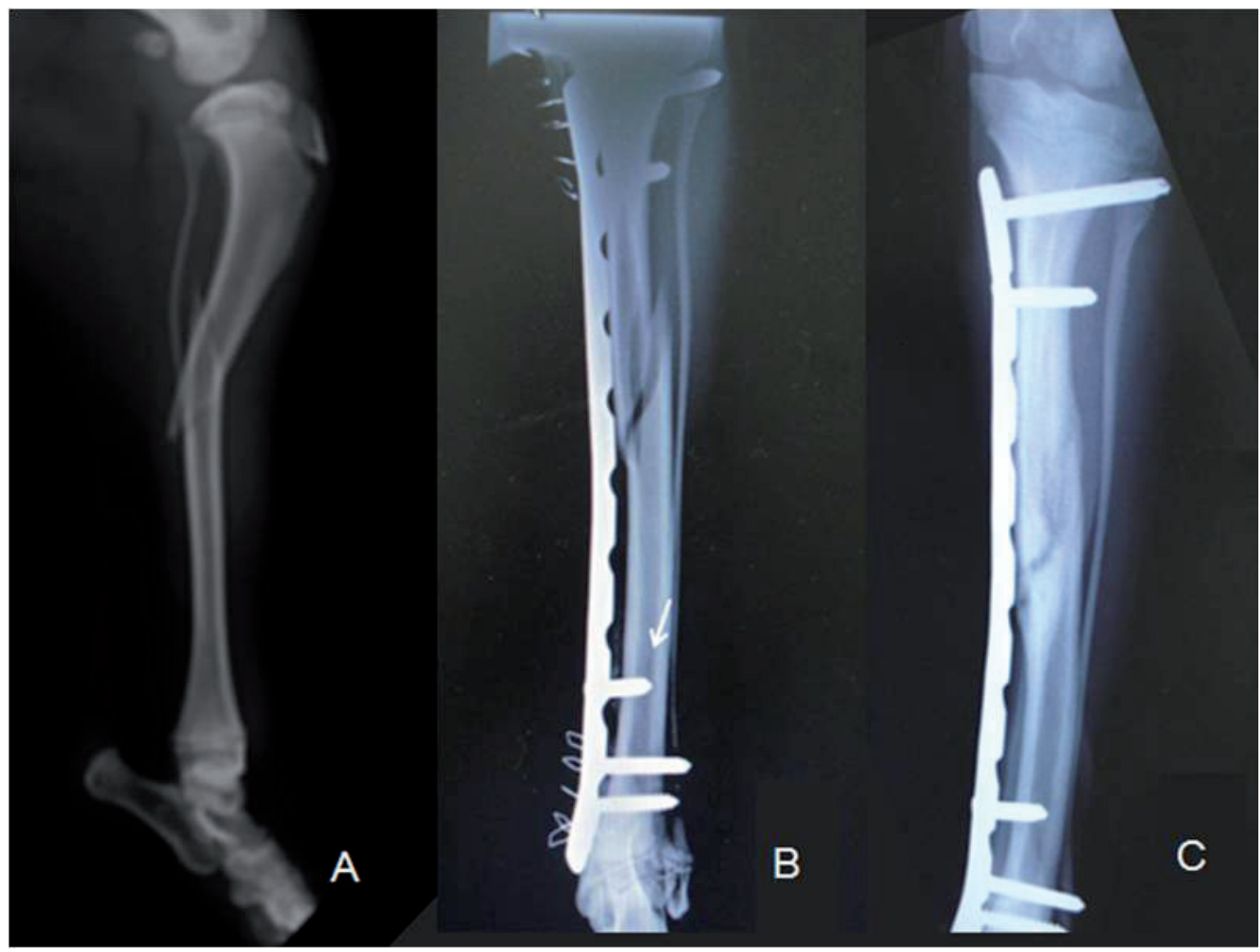

Figura 3 - Radiografias de um paciente canino com fratura de tíbia, $12 \mathrm{Kg}$ e quatro meses de idade. A. Fratura completa de tíbia. B - POB usada na fratura de tíbia por procedimento minimamente invasivo. Observar parafuso monocortical (seta). C - Um mês após o procedimento. Observar cicatrização quase completa e afastamento da placa em relação ao osso 
tura foi tratada com pino intramedular e placa bloqueada, sendo dois parafusos distais e quatro proximais.

\section{Técnica de placa bloqueada}

Após alinhamento dos fragmentos ósseos, a placa foi minimamente moldada com auxílio de retorcedor manual ou de mesa. Com instrumento específico push pull, a placa foi fixada a um dos fragmentos. $\mathrm{Na}$ sequência, perfurava-se o osso com auxílio de broca $2,8 \mathrm{~mm}$ para placa $3,5 \mathrm{~mm}$, aferia-se a profundidade do orifício com medidor de profundidade e o parafuso rosqueante era inserido com chave acoplada a torquímetro de 1,5 Newtons até prender se à placa. Após fixação dos parafusos, removia se o push pull e prendia-se o último parafuso.

Após a sutura de rotina, o membro foi imobilizado com bandagem de conforto do tipo Robert Jones. Todos os pacientes eram avaliados por meio de radiografias, no pós-operatório imediato. A prescrição pós-operatória padrão consistiu em administração de: dipirona (Dipirona sódica $500 \mathrm{mg}$, Laboratório Medley, São Paulo - SP, Brasil) na dose de $25 \mathrm{mg} / \mathrm{kg}$ a cada 08 horas por dez dias, carprofeno (Rimadyl 25 mg, 75 mg ou 100 mg, Laboratório Pfizer, São Paulo - SP, Brasil) na dose de $2,2 \mathrm{mg} / \mathrm{kg}$ a cada 12 por dez dias, cloridrato de tramadol (Cloridrato de tramadol 50mg, Laboratório Libbs Farm Ltda., São Paulo - SP, Brasil) na dose de $2 \mathrm{mg} / \mathrm{kg}$ a cada oito horas por cinco dias e cefalexina (Cefalexina $500 \mathrm{mg}$, Laboratório Medley, São Paulo - SP, Brasil) na dose de $30 \mathrm{mg} / \mathrm{kg}$ a cada 12 horas por dez dias.

O primeiro retorno foi agendado decorridos sete dias da cirurgia, para avaliação ortopédica, troca de bandagem e remoção de sutura cutânea e, na sequência, a cada 30 dias para avaliação ortopédica e realização de exames radiográficos controles até a consolidação óssea.

\section{Resultados e Discussão}

Os seis animais apresentados com luxação rádio-cárpica haviam sofrido queda de laje, que, associada ao peso elevado dos animais resultou na lesão carac- terística. A lesão leva frequentemente à impotência funcional, fato observado em todos os cães.

Todos os pacientes com luxação rádio-cárpica foram submetidos à artrodese com placa bloqueada. Nos pacientes acima de $40 \mathrm{~kg}$, utilizaram-se cinco parafusos no rádio, dois em carpo e três em metacarpo. Nos outros dois pacientes, utilizaram-se três parafusos no rádio, dois no carpo radial e dois no terceiro metacarpo. Os animais 01, 02, 03 e 04 apresentavam luxação unilateral. Todos evoluíram com claudicação leve em menos de um mês de pós-operatório e apoio normal em menos de dois meses. A consolidação óssea ocorreu com média de 3,5 meses e a placa foi removida aos 12 meses de pós-operatório.

Os animais 05 e 06 apresentavam luxação bilateral e ambos desenvolveram osteomielite bilateral. $\mathrm{O}$ animal 05 desenvolveu osteomielite após 45 dias, a qual foi tratada com resolução do caso após cinco meses e meio. A consolidação ocorreu em sete meses e após 1,5 anos, o paciente recebeu alta clínica. No animal 06, a osteomielite ocorreu aos quatro meses de pós-operatório e evoluiu para discoespondilite e óbito em 2,5 anos após a cirurgia. Em todos os pacientes, a placa foi removida após a consolidação. A remoção da placa é importante em áreas com pouca cobertura muscular e em casos de osteomielite, pois o implante pode perpetuar a infecçãa $0^{5,6}$.

Dentre os animais com fratura de fêmur, os números 07 e 08 foram apresentados logo após o trauma, porém, o animal número 09 já havia sido operado há dois meses, apresentava não união e pino rosqueado inserido na articulação. Os animais 07 e 08 foram tratados com pino intramedular e placa bloqueada. No primeiro, houve consolidação óssea em 45 dias, o pino intramedular foi removido e o paciente recebeu alta com recuperação completa da função do membro. No paciente 08 , houve consolidação em seis meses e retorno à deambulação normal. O animal 09 representava complicação com não união e osteomielite, foi submetido à osteossíntese de fêmur com placa 
bloqueada, sendo quatro parafusos distais e quatro proximais. A osteomielite foi tratada com antibiótico e houve resolução em quatro meses assim como a consolidação, semelhante aos resultados de Xiong et al. ${ }^{7}$ É possível obter consolidação mesmo em presença de osteomielite quando há estabilidade e compressão ${ }^{7,8}$. Em todos os casos, os implantes foram removidos como forma de evitar osteopenia e osteomielite ${ }^{5,6}$. Após fisioterapia, o animal 09 recebeu alta clínica com apoio normal.

Dos 13 animais submetidos à técnica de $\mathrm{POB}$, dois apresentavam luxação de patela. No animal 10 a consolidação foi observada em quatro meses. O paciente recebeu alta com deambulação satisfatória. $\mathrm{O}$ animal 11 , após o tratamento, apresentou deambulação satisfatória.

O animal 12 apresentou, ao exame radiográfico com 30 dias de pós-operatório, consolidação óssea sem sinais de claudicação. Um ano após, a placa foi removida como forma de prevenir complicações inerentes ao

\section{Referências}

1.FRIGG, R. Locking Compression Plate (LCP) An osteosynthesis plate based on the Dynamic Compression Plate and the Point Contact Fixator (PC-Fix). Injury, v. 32, p. 63-66, 2001. Supplement, 2.

2.HAALAND, P. J.; SJÖSTRÖM, L.; DEVOR, M.; HAUG A. Appendicular fracture repair in dogs using the locking compression plate system: 47 cases. Veterinary and Comparative Orthopaedics Traumatology, v. 22, n. 4, p. 309-315, 2009.

3. FERRIGNO, C. R. A.; ITO, K. C.; ROMANO, L.; DELLA NINA, M. I.; FERRAZ, V. C. M. Emprego clínico de placas ósseas bloqueadas em fraturas cominutivas de fêmur em cães. Primeiros relatos da utilização na ortopedia vete rinária nacional. In: CONGRESSO BRASILEIRO DE VETERINÁRIA, 2007, Santos, São Paulo, CD.

4. JIA, W.; ZHANG, C. H.; SHENG, J.; JIN, D.; CHENG, X.; CHEN, S.; ZENG, B. Free vascularized fibular grafting in combination with a locking plate for the reconstruction of a large tibial defect secondary to osteomyelitis in a child: a case report and literature review. Journal Pediatric Orthopaedics B, v. 19, n. 1, p. 66-70, 2010.

5. REIS, H. B.; SILVA, M. B.; OHARA, G.; SKAF, A. Y. Fratura da diáfise do úmero no adulto. Projeto Diretrizes: Associação Médica Brasileira e Conselho Federal de Medicina. Sociedade Brasileira de Ortopedia e Traumatologia. Associação Brasileira de Cirurgia da Mão. Colégio Brasileiro de Radiologia. Disponível em: <http://www.projetodiretrizes.org.br/7_volume/07Fratura_Dia.Ume.Adu.pdf $>$. Acesso em: 23 fev. 2011.

6. LABRONICI, P. J.; FRANCO, J. S.; SILVA, A. F. da; CABRAL, F. M. P.; SOARES, M. S.; LOURENÇO, P. R. B. implante ${ }^{4}$. Schwandt e Montavon ${ }^{11}$ também relataram sucesso com uso de POB em tíbias, assim como Haaland et al. ${ }^{2}$.

O animal 13 recuperou deambulação normal e a consolidação óssea ocorreu em cinco meses, resultados semelhantes aos de Reis et al. ${ }^{5}$. POB também é indicada para fraturas complexas ${ }^{1}$ como aquelas causadas por armas de fogo ${ }^{3,5}$.

\section{Conclusões}

Estudos clínicos e testes mecânicos comprovaram a eficiência das POB em termos de resistência e facilitação da cicatrização por não interferir no aporte vascular. $\mathrm{O}$ implante pode ser utilizado na medicina veterinária e a técnica exige planejamento pré-operatório, especialmente na sequência de aplicação dos diferentes tipos de parafusos. Apresenta custo elevado, mas confere estabilidade rígida ao foco de fratura e minimiza a possibilidade de perda prematura da interface parafuso e osso.

T.; HOFFMANN, R.; FERNANDES, H. J. A.; REIS, F. R. Tratamento das fraturas distais da tíbia. Acta Ortopédica Brasileira, v. 17, n. 1, p. 40-45, 2008.

7.XIONG, Y.; ZHAO, Y.; WANG, Z.; DU, Q.; CHEN, W.; WANG, A. Comparison of a new minimum contact locking plate and the limited contact dynamic compression plate in an osteoporotic fracture model. International Orthopaedics, v. 33, n. 5, p. 1415-1419, 2008.

8. OH, C.; KIM, J.; BYUN, Y.; OH, J.; KIM, J.; KIM, S.; PARK, B.; LEE, H. Minimally invasive plate osteosynthesis of subtrochanteric femur fractures with a locking plate: a prospective series of 20 fractures. Archives of Orthopaedic Trauma Surgery, v. 129, n. 12, p. 1659-1665, 2009.

9. AGUILA, A. Z.; MANOS, J. M.; ORLANSKY, A. S.; TODHUNTER, R. J.; TROTTER, E. J.; VAN DERMEULEN, M. C. H. In vitro biomechanical comparison of limited contact dynamic compression plate and locking compression plate. Veterinary and Comparative Orthopaedics Traumatology, v. 18, n. 4 , p. 220-226, 2005.

10.GOH, G. S.; SANTONI, B. G.; PUTTLITZ, C. M.; PALMER, R. $\mathrm{H}$. Comparison of the mechanical behaviors of semicontoured, locking plate-rod fixation and anatomically contoured, conventional plate-rod fixation applied to experimentally induced gap fractures in canine femora. American Journal of Veterinary Research, v. 70, n. 1, p. 23-29, 2009.

11.SCHWANDT, C. S.; MONTAVON, P. M. Locking compression plate fixation of radial and tibial fractures in a young dog. Veterinary and Comparative Orthopaedics Traumatology, v. 18, n. 3, p. 194-198, 2005. 